Chirurg 2013 $\cdot 84: 997$

DOI 10.1007/s00104-013-2632-7

Online publiziert: 13. Oktober 2013

(c) Springer-Verlag Berlin Heidelberg 2013

H. Dralle $\cdot$ I. Satiroglu

Universitätsklinik für Allgemein-, Viszeral- und Gefäßchirurgie, Medizinische Fakultät,

Universitätsklinikum Halle (Saale)

\title{
Malignitätsrisiko kleiner hormoninaktiver neuroendokriner Pankreastumoren
}

\section{Diskussion und Fazit}

Im Samsung Medical Centre Seoul, Korea, wurden zwischen 1994 und 2010 insgesamt 125 Patienten wegen eines HINEPT offen (112) oder laparoskopisch (13) pankreasreseziert. Die Patientenzahl zwischen 2005 und 2010 war doppelt so hoch wie zwischen 2000 und 2005 (70 vs. 36). Die mediane Nachbeobachtungszeit betrug 31,5 (3,6-145,1) Monate.

\section{Ergebnisse}

Bei 76 Patienten (61\%) mit HINEPT handelte es sich um einen Zufallsbefund, die übrigen Patienten waren abdominell symptomatisch, nur 10 Patienten hatten pankreasspezifische Befunde (Ikterus, palpabler Tumor). Bei 65 Patienten (52\%) waren die Tumoren $2 \mathrm{~cm}$ oder kleiner ( $<1 \mathrm{~cm}: \mathrm{n}=14 ; 1-2 \mathrm{~cm}: \mathrm{n}=51)$.

Bei den Tumoren $\leq 1 \mathrm{~cm}$ lagen nur in 2 Fällen gut differenzierte neuroendokrine Karzinome vor, in keinem Fall Lymphknoten- oder Fernmetastasen; Rezidive oder ein tumorbedingtes Versterben wurden nicht beobachtet. Bei Patienten mit Tumoren zwischen 1 und $2 \mathrm{~cm}$ wurden dagegen in $24 \%$ gut oder wenig differenzierte neuroendokrine Karzinome diagnostiziert, ein Patient mit einem 1,2 cm großen Tumor hatte Lymphknotenmetastasen, 3 Patienten entwickelten Rezidive in der Leber, die bei den beiden Patienten mit wenig differenzierten Karzinomen innerhalb 6 Monaten nach primärer Duodenopankreatektomie auftraten.

\section{$1 \mathrm{~cm}$ Größe beobachtet werden können, bei Größenzunahme oder initialer Größe über $1 \mathrm{~cm}$ jedoch unter kurativer Intention eine Pankreas- resektion zu präferieren ist.}

\section{Korrespondenzadresse}

Prof. Dr. Dr. h.c. H. Dralle

Universitätsklinik für Allgemein-, Viszeralund Gefäßchirurgie, Medizinische Fakultät, Universitätsklinikum Halle (Saale), Ernst-Grube-Str. 40, 06097 Halle (Saale) henning.dralle@uk-halle.de

\section{Einhaltung ethischer Richtlinien}

Interessenkonflikt. H. Dralle und I. Satiroglu geben an, dass kein Interessenkonflikt besteht.

Dieser Beitrag beinhaltet keine Studien an Menschen oder Tieren.

\section{Literatur}

1. Bettini R, Partelli S, Boninsegna L et al (2011) Tumor size correlates with malignancy in nonfunctioning pancreatic endocrine tumor. Surgery 150:75-

- Das tumorbiologische Spektrum bei HINEPT ist weitgehend abhängig von der Primärtumorgröße; wenig differenzierte und G3-Tumoren fanden sich nicht bei Tumoren kleiner $1 \mathrm{~cm}$.

- Bei Primärtumoren zwischen 1 und $2 \mathrm{~cm}$ waren ca. ein Viertel gut oder sogar gering differenzierte neuroendokrine Karzinome mit Potenzial zur primären oder sekundären Metastasierung; ein Patient dieser Gruppe verstarb am Tumor.

- Die vorliegenden Ergebnisse sprechen dafür, dass HINEPT unter
2. Kim MJ, Choi DW, Choi SH et al (2012) Surgical strategies for non-functioning pancreatic neuroendocrine tumours. Br J Surg 99:1562-1568 